\title{
Transport Parameters and Breakdown Voltage Characteristics of Gas Mixture Representing Martian Atmosphere and Its Constituents
}

\author{
Received: 10.02.2021 \& Accepted: 26.03.2021 \\ M. Radmilović-Radjenović* And B. Radjenović \\ Institute of Physics, University of Belgrade, Pregrevica 118, 11080 Belgrade, Serbia \\ Doi: 10.12693/APhysPolA.139.698 \\ *e-mail: marija@ipb.ac.rs

\begin{abstract}
This paper reports on transport parameters and the breakdown voltage curves of the gases included in the composition of the Martian atmosphere. The planet Mars has a thin, carbon dioxide-rich atmosphere, with an atmospheric volume of less than $1 \%$ of Earth's. The understanding of electrical discharge processes occurring in the Martian atmosphere and potential hazards due to arcing on landers and rovers becomes increasingly important for designing space-flight hardware. Although local convective events known as dust devils occur daily, the focus was put on the electric discharge which causes problems even in dust-free atmospheres. Ionization coefficients, electron energy distribution functions, and mean energy for the Mars gas mixture and its constituents were calculated by Bolsig + , a numerical solver for the Boltzmann equation. It was found that Mars mixture and carbon dioxide as a majority component are similar and not affected by the addition of other components. The results of this study indicate that flight electronics should be designed not to exceed an absolute potential difference of more than $511 \mathrm{~V}$ when operating in the Martian atmosphere.
\end{abstract}

topics: atmosphere, mixture, discharges, arcing

\section{Introduction}

In recent years, we have witnessed numerous space missions to the surface of Mars [1-4]. One of the risks to rovers, landers, and instrumentation at the Martian surface is electrification a fundamental process in planetary atmospheres causing failure of more than $60 \%$ of landing attempts $[5,6]$. In the Mars-like atmosphere, winds bringing sand and dust develop near-surface electric fields that result in either Townsend dark discharge or normal glow discharge which appears as a dim glow [7]. Studies of mechanisms and conditions that could lead to the discharge are essential for designing space-flight hardware, as well as understanding electrical discharge processes occurring in the Martian atmosphere [8]. Designs of electrical components on spacecraft are based on estimations by either using breakdown voltage curves for carbon dioxide or argon, but neither of these is representative of the breakdown characteristics of Mars atmosphere.

Mechanisms of electrical discharges on Mars have been the subject of numerous papers. According to Mills [9], glow discharges occur within the lifted dust in the Martian atmosphere. Farrell and coworkers suggested the existence of different types of discharges from a filamentary discharge similar to lightning on Earth, to glow discharge around the dust cloud [10]. Aplin [11] has studied the effect of electrified dust clouds on the existence and properties of a global electrical circuit on Mars. Chemical effects as an important aspect of electrical activity within dust events on the surface of Mars with potential importance for astrobiology are considered in [12-15]. Recently, Boltzmann Analysis has been applied to determine electron swarm parameters and limiting electrical field during Martian dust storm season [16].

Lightning on Mars takes place within condensate clouds, from charging to breakdown, to propagation and emission of radiation in various bands of the electromagnetic spectrum [17]. While the specific nature of the charging processes taking place in different planetary atmospheres may differ, the overall mechanism is similar. The electric fields become strong enough to exceed the local breakdown value, leading to the generation of streamers, leaders, and finally discharge channels.

The basic assumption in general studies is that the electrical activity on Mars is similar to that in Earth's atmosphere [18]. Although the Martian atmosphere is the closest in terms of its meteorology and climate to that of Earth, it is much thinner and cold, rich in dust aerosols [19, 20]. Gas mixture representing the Martian atmosphere is composed of $95.3 \% \mathrm{CO}_{2}, 2.7 \% \mathrm{~N}_{2}, 1.6 \% \mathrm{Ar}, 0.13 \% \mathrm{O}_{2}$, $0.07 \% \mathrm{CO}, 0.03 \%$ water vapor, and the rest trace 
gases [20]. Since the Martian atmosphere is not pure carbon dioxide but contains a small amount of argon, the necessity of studying the breakdown voltages in a simulated Martian atmosphere was reported [21].

In this paper, we study breakdown voltage curves for a gas mixture representative of the Martian atmosphere and its constituents by using Bolsig + code [22-24] with the cross-sections data taken from [25]. Since it is composed mostly of $\mathrm{CO}_{2}$, it was shown that the breakdown voltage curves of Mars's mixture gas and pure $\mathrm{CO}_{2}$ are comparable. Although dust plays an important role in Martian atmospheric electricity, we focus on the electric discharge in dust-free atmospheres only to emphasize that even within a spacecraft, at places not exposed to dust, an electric discharge can be an issue at the Martian atmospheric pressure range. Despite the limitations of this code, the results of calculations are in good agreement with the results obtained by using expressions and appropriate parameters taken from [26] and data provided in [27-29].

\section{Calculation method}

Transport parameters associated with the Martian atmosphere have been determined by using Bolsig +, a user-friendly computer program for solving the Boltzmann equation (BE) of electrons in weakly ionized gases in a uniform electric field. The electron energy distribution function (EEDF) and the transport coefficients are calculated from the fundamental collision cross-section data by solving BE [22]:

$$
\frac{\partial f}{\partial t}+\boldsymbol{v} \cdot \nabla f-\frac{e}{m} \boldsymbol{E} \cdot \nabla_{v} f=C(f),
$$

where $f$ is the electron distribution, $\boldsymbol{v}$ is the velocity coordinate, $e$ is the elementary charge, $m$ is the electron mass, $\boldsymbol{E}$ is the electric field, while $\nabla_{v}$ is the velocity-gradient operator. On the right-hand side of (1), the function $C$ represents the rate of change in $f$ due to collisions. To solve the $\mathrm{BE}$, one has to simplify it, such as assuming spatial uniformity of the electric field and the collision probabilities. The electron distribution $f$ becomes then symmetric in velocity space around the electric field direction, while in position space, it may vary only along the field direction. Using spherical coordinates in velocity space, (1) can be rewritten [22]. Namely,

$$
\begin{aligned}
& \frac{\partial f}{\partial t}+v \cos (\theta) \frac{\partial f}{\partial z} \\
& -\frac{e E}{m}\left(\cos (\theta) \frac{\partial f}{\partial v}+\frac{\sin ^{2}(\theta)}{v} \frac{\partial f}{\partial \cos (\theta)}\right)=C(f),
\end{aligned}
$$

where electron distribution $f$ depends on the magnitude of the velocity $v$, the angle between the velocity and the field direction $\theta$, the position along the field direction $z$, and the time $t$. Further, $\theta-$ dependence can be simplified by the classical twoterm approximation (TTA).
The basic idea under the TTA is the representation of the electron distribution $f$ by the first two terms of an expansion in spherical harmonics in velocity space [22]:

$$
f(v, \cos (\theta), z, t)=f_{0}(v, z, t)+f_{1}(v, z, t) \cos (\theta),
$$

where $f_{0}$ and $f_{1}$ are the isotropic and anisotropic parts, respectively. If the electric field is constant, the solution for the EEDF can be expressed as a function of the reduced electric field $E / N$ (the ratio of the electric field to the gas density).

In our calculations, we assumed that the Martian atmosphere is composed of $95.3 \% \mathrm{CO}_{2}, 2.7 \%$ $\mathrm{N}_{2}, 1.6 \% \mathrm{Ar}$, and $0.13 \% \mathrm{O}_{2}$. Calculations were performed for Mars's gas mixture and its constituents, for the reduced electric fields ranging from 50 to $1000 \mathrm{Td}$. In the calculations, cross-sections for 58 elastic and inelastic electron-impact collisional processes have been used. The sets of cross-sections for carbon dioxide, nitrogen, argon, and oxygen include $13,25,3$, and 17 processes, respectively. The ionization coefficients, electron energy distribution functions, and the breakdown voltage curves have been determined.

\section{Results}

Results of the TTA calculation for the ionization coefficients $\alpha / N$ for the gas mixture representing and gases included in the Martian atmosphere are shown in Fig. 1a. The ionization coefficient of the Martian atmosphere (down triangles) strongly resembles the ionization coefficient of carbon dioxide (up triangles). The ionization coefficient of oxygen is similar to those of carbon dioxide and Mars. However, the ionization coefficients of argon and nitrogen are systematically higher and lower, respectively. Results for $\mathrm{N}_{2}, \mathrm{O}_{2}$, and $\mathrm{Ar}$ are fitted with a semi-empirical formula [26]:

$$
\frac{\alpha}{N}=\sum_{i} A_{i} \mathrm{e}^{-B_{i} /(E / N)},
$$

where $E / N$ is the reduced field (electric field to the gas number density ratio), while $A_{i}$ and $B_{i}$ are the constants. Results for the gas mixtures are fitted by the expression taken from [26]:

$$
\left[\frac{\alpha}{N}\left(\frac{E}{N}\right)\right]_{m}=\sum_{z} x_{z}\left[\frac{\alpha}{N}\left(\frac{E}{N}\right)\right]_{z},
$$

where $x_{z}$ is the label for the fraction of the gas $z$ in the mixture (i.e., $\sum_{z} x_{z}=1$ ). A good agreement between TTA calculations (symbols) and model values based on (4) and (5) (lines) has been achieved. For comparison, results of TTA calculations for carbon dioxide (red up triangles) agree well with the data published in [27] (open-up triangles). Linearized representation of $\ln (\alpha / N)$ versus $N / E$ is depicted in Fig. 1b. As expected, by using this representation, the behavior of the data points is 

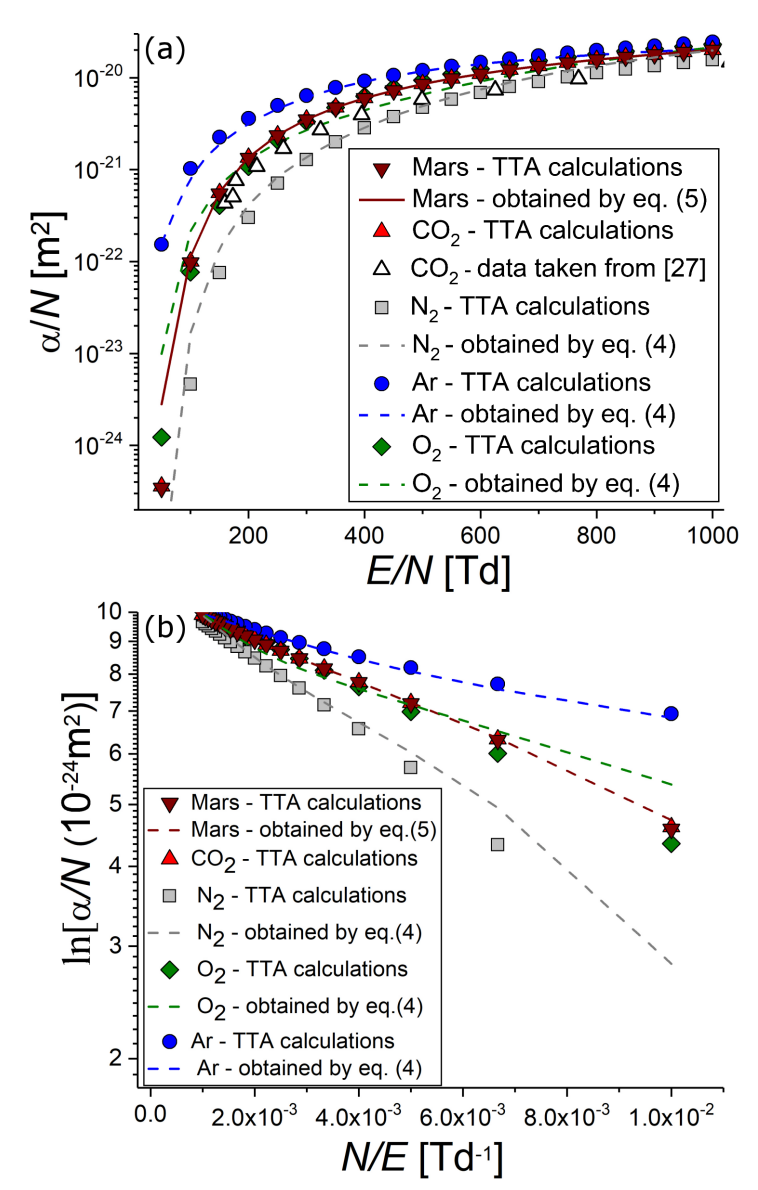

Fig. 1. Results of: (a) density-normalized first ionization coefficient as a function of $E / N$ and (b) corresponding linearized representation of $\ln (\alpha / N)$ versus $N / E$ for the Martian atmosphere gas mixture and its constituents. Solid symbols and dashed lines represent results of TTA calculations and with (4), (5), respectively. Experimental data [27] are shown by open-up triangles.

almost linear [28]. Also, the Martian atmosphere mixture follows the behavior of the $\mathrm{CO}_{2}$ as a majority component.

Figure 2 demonstrates the changes in the slope of the EEDFs at different values of $E / N$. For all $E / N$ values, EEDFs for $\mathrm{CO}_{2}$ and carbon dioxide are similar. At low values of the reduced field $E / N$, large differences between EEDFs can be observed. At higher values of the reduced field, however, the EEDFs for all constituents are very similar, except for the carbon dioxide and the Martian atmosphere mixture which could be attributed to the non-proper extrapolation of its cross-section data. Electron kinetics is strongly affected by the electric field and also by inelastic collisions due to the inefficiency of elastic collisions in the energy transfer. Therefore, the EEDFs at the same reduced field $E / N$ for individual gases $\left(\mathrm{N}_{2}, \mathrm{Ar}\right.$, and $\left.\mathrm{O}_{2}\right)$ and the mixture are quite different. Consequently, mean electron energies for constituent gases and the mixture may be considerably different, as one
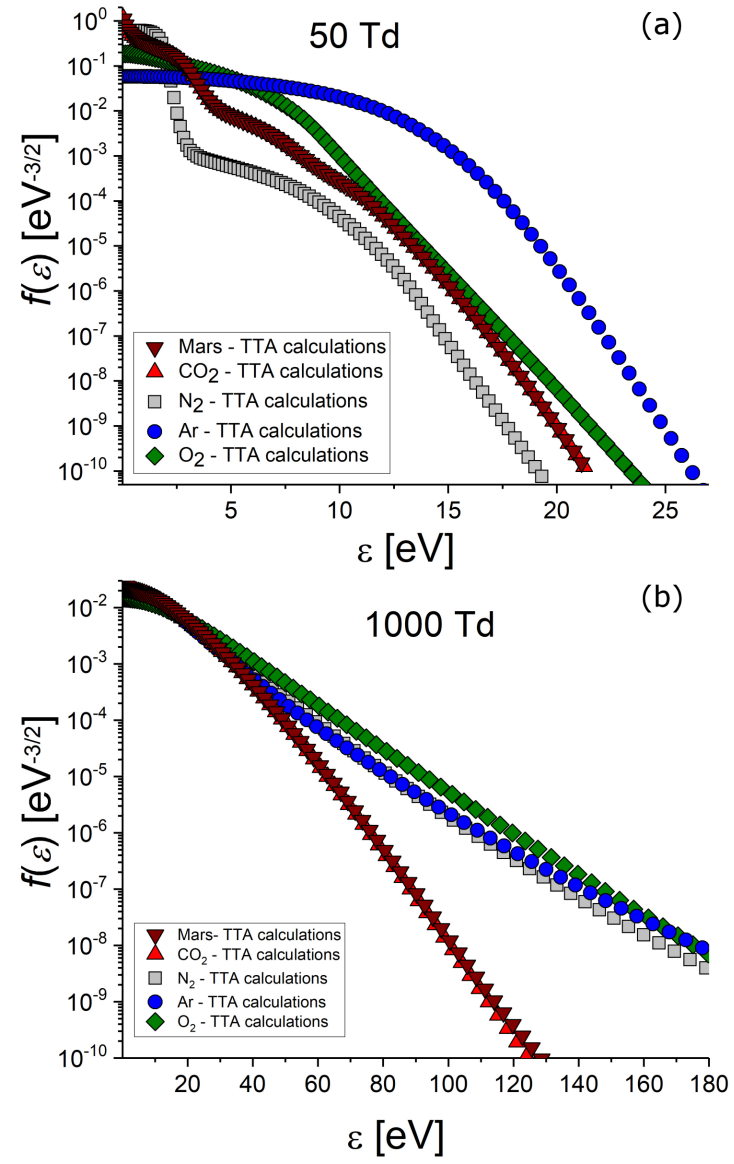

Fig. 2. TTA calculation results for the electron energy distribution function of the individual gases (carbon dioxide, nitrogen, oxygen, and argon) and their composition representing the Martian atmosphere at the reduced electric field of: (a) $50 \mathrm{Td}$ and (b) $1000 \mathrm{Td}$.

can see in Fig. 3. This holds for $\mathrm{Ar}$ and $\mathrm{N}_{2}$ all over the range of $E / N$. The mean energy of $\mathrm{O}_{2}$ significantly differs from that for the mixture at low and high values of $E / N$, while it is similar for medium $E / N$.

The dependence of the ionization coefficients on the mean energy is presented in Fig. 4. If we compare $\alpha / N$ values at the same energy, it is obvious that for the lower mean electron energies ionization is quite different in the mixture and the individual gases due to different electron energy distributions. In this range of energies, the ionization of the Mars mixture and carbon dioxide prevails over the ionization of oxygen, nitrogen, and argon.

To determine the breakdown voltages, the calculated values for the ionization coefficient were fitted according to the empirical expression $\alpha_{\text {eff }} / p=A \mathrm{e}^{-B p / E}$. Determined parameters $A$ and $B$ are listed in Table I and used in the expression for the breakdown voltage $V_{\mathrm{B}}[29]$ :

$$
V_{\mathrm{B}}=-\frac{B p d}{\ln \left(\frac{\ln (Q)}{A p d}\right)},
$$




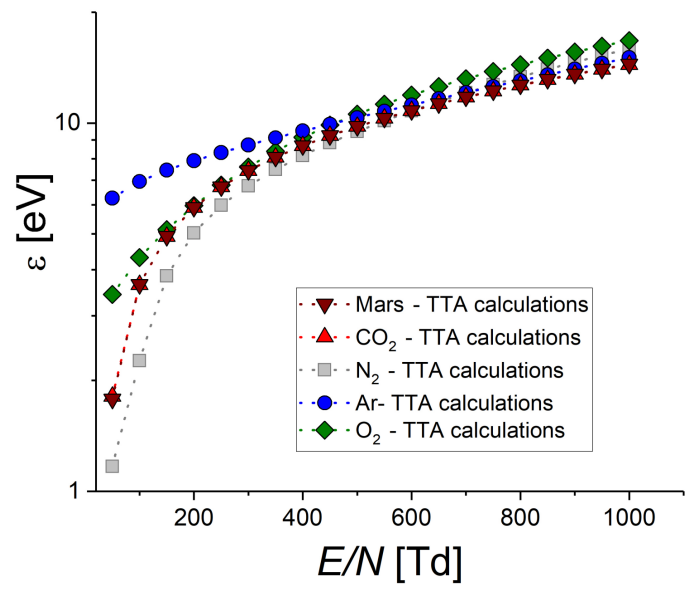

Fig. 3. The dependence of electron mean energies $\varepsilon$ on the reduced field $E / N$ in carbon dioxide (red up triangles), nitrogen (gray squares), argon (blue circles), oxygen (green diamonds) and its mixture (brown down triangles).

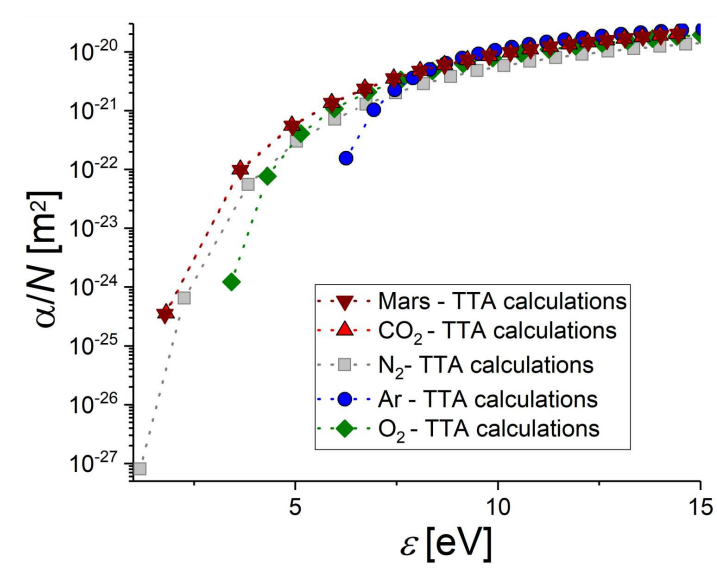

Fig. 4. TTA calculation results for the ionization coefficients as a function of mean electron energy $\varepsilon$ for the Martian atmosphere and constituent gases in the mixture separately.

where $p$ is the pressure, $d$ is the distance, while $Q=10^{4}$ is estimated from the avalanche criteria [29]. The classical Paschen curve has a minimum - calculated from the condition $\mathrm{d} V_{\mathrm{B}} / \mathrm{d}(p d)=0[29]$. As a result, one obtains

$$
V_{\min }=\frac{e B}{A} \ln (Q), \quad(p d)_{\min }=\frac{e}{A} \ln (Q) .
$$

The breakdown voltage curves of gas mixture representing the Martian atmosphere and its constituents are shown in Fig. 5. $\mathrm{CO}_{2}$ and Mars gas have comparable curves indicating that the addition of small amounts of $\mathrm{N}_{2}, \mathrm{O}_{2}$, and Ar does not considerably alter the discharge potential. The Martian gas breakdown voltages are equal to or slightly smaller than the $\mathrm{CO}_{2}$ voltages due to the known lower breakdown potentials of nitrogen and argon, present in small concentrations. The experimentally recorded breakdown voltage curve [30] is shown by crosses. Discrepancies between the experimentally recorded

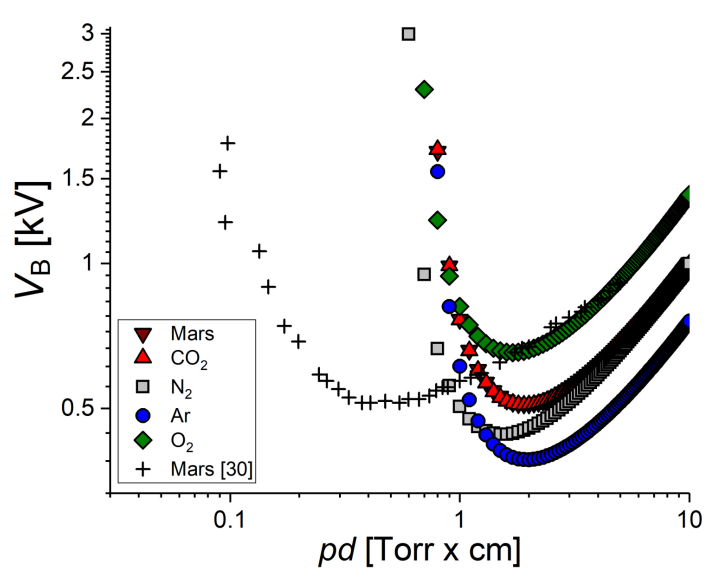

Fig. 5. The breakdown voltage $V_{\mathrm{B}}$ versus the $\mathrm{pd}$ product (the pressure times the distance) for the gases included in the mixture representing the Martian atmosphere. Crosses correspond to the experimental data taken from [30].

TABLE I

Exponential approximation coefficients $(A$ and $B)$ from the calculated values of the ionization coefficient by fitting with $\alpha_{\text {eff }}(E)=A p \mathrm{e}^{-B p / E}$. The minima of $V_{\min }$ and $(p d)_{\min }$ are calculated according to $(7)$.

\begin{tabular}{l|c|c|c|c}
\hline \hline Coefficient & $\begin{array}{c}\text { Ref. [29] } \\
\text { (calc.) }\end{array}$ & $\begin{array}{c}\text { Ref. [31] } \\
\text { (calc.) }\end{array}$ & $\begin{array}{c}\text { Ref. [30] } \\
\text { (exper.) }\end{array}$ & $\begin{array}{c}\text { this } \\
\text { study }\end{array}$ \\
\hline$A[1 /(\mathrm{cm}$ Torr)] & 33.44 & 7.23 & - & 13.04 \\
$B[\mathrm{~V} /(\mathrm{cm}$ Torr)] & 430.07 & 178 & - & 265.8 \\
$V_{\text {min }}[\mathrm{V}]$ & 356.1 & 462 & 514 & 511 \\
$(p d)_{\text {min }}[$ Torr cm] & 3.9 & 2.6 & 0.6 & 1.9
\end{tabular}

(crosses) [30] and our calculation results (down triangles) for the Martian breakdown voltage curves can be attributed to different experimental and calculation conditions. Although the same gas mixture representing the Mars atmospheric composition is used in both measurements and calculations, the pressure and the gap were different. Also, the procedures of the breakdown voltage determination are different. In the experiment, starting from $100 \mathrm{~V}$, the applied voltage was increased in increments of $50 \mathrm{~V}$ until the current reached about $500 \mathrm{~mA}$. On the other hand, in calculations, the breakdown voltage curve is determined with (6) and the avalanche criteria $Q=10^{4}$.

In Table I, values of the $A$ and $B$ parameters, the breakdown voltage and the pd product value at the minimum corresponding to the Matian atmosphere obtained in this study are compared with the data taken from [29-31]. Our result for the minimum breakdown voltage of $511 \mathrm{~V}$ agrees well with the measured value of $514 \mathrm{~V}$ [30] but differs from the calculated values of around $359 \mathrm{~V}$ [29] and $462 \mathrm{~V}$ [31], respectively. Our result for the pd value at the minimum agrees better with the data found in [31]. The difference between our BOLSIG + result and the experimental data [30] for 
the position of the minimum of the breakdown voltage curve is around $68 \%$. The experimental value for the pd value at the minimum of the breakdown voltage curve disagrees from the calculated values taken from [29] and [31] around 77\% and 84\%, respectively.

\section{Conclusions}

In this paper, transport parameters and the breakdown voltage curves of the gas mixture representing the Martian atmosphere and its constituents have been presented and analyzed. Calculations were performed for the temperature and atmospheric composition of Mars by using Bolsig+ [22]. The results of TTA calculations for the ionization coefficients are in good agreement with the available data [27] and the theoretical predictions [26]. The obtained results provide an insight into similarities and differences between the transport parameters that correspond to the individual gases and their mixture. It was shown that the difference between parameters of the Martian atmosphere and carbon dioxide is negligible which can be explained by a small abundance of their constituent gases as compared to the abundances of the carbon dioxide. Also, it seems that $\mathrm{CO}_{2}$ dictates the breakdown voltage curve of the Martian atmosphere and that relatively low concentrations of other gases do not affect it substantially. The results are validated by comparison with the measured Paschen curve for a gas mixture representative of the Martian atmosphere [30]. The $A$ and $B$ coefficients derived from the exponential fit accurately predict the minimum breakdown voltage of $511 \mathrm{~V}$ which agrees with the experimental data found in the literature [30]. For the position of the minimum at the breakdown voltage curve, however, our BOLSIG + result disagrees with the measured value by around $68 \%$. On the other hand, differences between our calculated value for the minimum breakdown voltage and numerical solutions taken from [29] and [31] are around 9\% and $30 \%$, respectively. For the pd value at the minimum, the differences between our result and calculated values from [29] and [31] are around 51\% and $27 \%$.

\section{Acknowledgments}

The authors acknowledge funding provided by the Institute of Physics Belgrade, through the grant by the Ministry of Education, Science and Technological Development of the Republic of Serbia.

\section{References}

[1] J.B. Garvin, O. Figueroa, F.M. Naderi, Astrobiology 1, 439 (2001).

[2] M. La Duc, W. Nicholson, R. Kern, K. Venkateswaran, Environ. Microbiol. 5, 977 (2003).
[3] S.W. Squyres, R.E. Arvidson, J.F. Bell et al., Science 306, 1698 (2004).

[4] V.F. Krapivin, C.A. Varotsos, J. Christodoulakis, Earth Moon Planets 118, 1 (2016).

[5] G. Horneck, G. Reitz, P. Rettberg, C. Baumstark-Khan, R. Gerzer, Adv. Space Res. 31, 87 (2003).

[6] A.G.M. Inza, G. Lopez-Reyes, Mars Exploration - a Step Forward, IntechOpen, London, 2020.

[7] T. Ogliore, "Electricity in Martian Dust Storms Helps to Form Perchlorates", Washington University in St. Louis, St. Louis 2018.

[8] S. Falcinelli, Acta Phys. Pol. A 131, 112 (2017).

[9] A.A. Mills, Nature 268, 614 (1977).

[10] W. Farrell, M. Kaiser, M. Desch et al., J. Geophys. Res. 104, 3795 (1999).

[11] K. Aplin, Surv. Geophys. 27, 63 (2006).

[12] S. Osman, Z. Peeters, M.T. La Duc, R.L. Mancinelli, P. Ehrenfreund, K. Venkateswaran, Appl. Environ. Microbiol. 74, 959 (2008).

[13] A.G. Fairén, V. Parro, D. Schulze-Makuch, L. Whyte, Astrobiology 18, 101 (2018).

[14] A. G.Fairén, D. Schulze-Makuch, L. Whyte, V. Parro, A. Pavlov, J. GómezElvira, A. Azua-Bustos, W. Fink, V. Baker, Adv. Space Res. 63, 1491 (2019).

[15] T. Fornaro, A. Boosman, J.R Brucato, I.L. Ten Kate, S. Siljeström, G. Poggiali, A. Steele, R.M. Hazen, Icarus 313, 38 (2018).

[16] M.S. Dincer, S.S. Tezcan, H. Duzkaya, S. Dince, Acta Phys. Pol. A 138, 368 (2020).

[17] Y. Yair, Adv. Space Res. 50, 293 (2012).

[18] R.G. Harrison, E. Barth, F. Esposito, J. Merrison, F. Montmessin, Space Sci. Rev. 203, 299 (2016).

[19] C. Gebhardt, A. Abuelgasim, Prog. Earth Planet Sci. 6, 31 (2019).

[20] D. Banfield, A. Spiga, C. Newman et al., Nat. Geosci. 13, 190 (2020).

[21] H.L.K. Manning, I.L. ten Kate, S.J. Battel, P.R. Mahaffy, Adv. Space Res. 46, 1334 (2010).

[22] G.J.M. Hagelaar, L.C. Pitchford, Plasma Sources Sci. Technol. 14, 722 (2005).

[23] S.S. Tezcan, H. Duzkaya, S. Dincer, M.S. Dincer, Acta Phys. Pol. A 135, 495 (2019).

[24] G. Hagelaar, Electron Boltzmann equation solver Bolsig + , ver. 12/2019. 
[25] Plasma Data Exchange Project, LXCat.

[26] D. Marić, M. Radmilović-Radjenović, Z.Lj. Petrović, Eur. Phys. J. D 35, 313 (2005).

[27] M. Vass, I. Korolov, D. Loffhagen, N. Pinhão, Z. Donkó, Plasma Sources Sci. Technol. 26, 065007 (2017).

[28] A.R. Petri, J.A.C. Gonçalves, A. Mangiarotti, S. Botelho, C.C. Bueno, Nucl. Instrum. Methods Phys. Res. A 849, 31 (2017).
[29] J.A. Riousset, in: AGU Fall Meeting 2016, San Francisco, 2016, AE21A-08.

[30] C.I. Calle, P.J. Mackey, M.D. Hogue, M.R. Johansen, J.D. Kelley, J.R. Phillips III, J.S. Clements, J. Electrostat. 71, 254 (2013).

[31] Ch. Helling, M. Jardine, C. Stark, D. Diver, Astrophys. J. 767, 136 (2013). 